\title{
Long-term expression of rAAV2-hIL15 enhances immunoglobulin production and lymphokine-activated killer cell-mediated human glioblastoma cell death
}

\author{
GIOU-TENG YIANG ${ }^{1,2}$, RUEY-HWANG CHOU ${ }^{3,4}$, WEI-JUNG CHANG ${ }^{3}$, \\ CHYOU-WEI WEI ${ }^{5}$ and YUNG-LUEN YU ${ }^{3,4}$

\begin{abstract}
${ }^{1}$ Department of Emergency Medicine, Tzu Chi University, Hualien 970; ${ }^{2}$ Department of Emergency Medicine, Buddhist Tzu Chi General Hospital, Taipei Branch, New Taipei $231 ;{ }^{3}$ Graduate Institute of Cancer Biology and Center for Molecular Medicine, China Medical University, Taichung 404;

${ }^{4}$ Department of Biotechnology, Asia University, Taichung $413 ;{ }^{5}$ Department of Nutrition,
\end{abstract} \\ Master Program of Biomedical Nutrition, Hungkuang University, Shalu, Taichung 433, Taiwan, R.O.C
}

Received July 18, 2012; Accepted December 14, 2012

DOI: $10.3892 / \mathrm{mco} .2013 .60$

\begin{abstract}
Glioblastoma is the most aggressive primary brain tumor and its prognosis remains poor despite different treatment modalities including surgery, radiotherapy and chemotherapy. Therefore, more useful treatments for glioblastoma patients are required. Human interleukin 15 (hIL15) is an immunomodulator that has antitumor activities. hIL15 combined with gene therapy method is also currently under cosideration as a treatment option. Since recombinant adenoassociated virus serotype 2 (rAAV2) with low immunogenicity and long-term gene expression in human clinical trials has been demonstrated, rAAV2 encoding hIL15 (rAAV2-hIL15) were used to inhibit human glioblastoma growth in the present study. rAAV2-hIL15, which is able to express IL15 protein with bioactivity, was successfully produced and purified. Data of this study demonstrated that the long-term expression of rAAV2-hIL15 enhances immunoglobulin (Ig) production and the cytotoxic activity of lymphokine-activated killer (LAK) cells. In addition, results of the present study showed that rAAV2-hIL15 delays tumor growth on a xenografted human glioblastoma mice model. Taken together, these results indicated that rAAV2-hIL15 constitutes a powerful and potent gene immunotherapy method for human glioblastoma treatment.
\end{abstract}

Correspondence to: Dr Yung-Luen Yu, Graduate Institute of Cancer Biology and Center for Molecular Medicine, China Medical University, Taichung 404, Taiwan, R.O.C

E-mail: ylyu@mail.cmu.edu.tw

Dr Chyou-Wei Wei, Department of Nutrition, Master Program of Biomedical Nutrition, Hungkuang University, Shalu, Taichung 433, Taiwan, R.O.C

E-mail:wcwnina@gate.sinica.edu.tw

Key words: glioblastoma, recombinant adeno-associated virus serotype 2, human interleukin 15 , lymphokine-activated killer cell, immunoglobulin

\section{Introduction}

Human glioblastoma is one of the most malignant brain tumors. Currently, the traditional methods used to treat glioblastoma include surgery, chemotherapy, radiotherapy and a combination of these methods (1). However, the mean survival time of glioblastoma patients is one year and most patients do not survive more than two years in the clinical course (1). Therefore, more useful treatments for glioblastoma patients are required, thus a number of gene therapies for tumor treatment have been suggested (2-4).

Human interleukin 15 (hIL15) is a $14-15 \mathrm{kDa}$ immunostimulatory cytokine that is able to induce cell proliferation and T-cell differentiation (5-7). A previous study demonstrated that immunotherapy with lymphokine-activated killer (LAK) cell cytotoxic activity has been widely used for breast, lung, ovary and pancreatic tumor treatment in phase II clinical trials (8). Due to the short half-life of IL15, repeated daily injection is required when employing IL15 administration, which is not convenient for human cancer treatments (9-11). Therefore, authors of previous studies suggested that replacing daily administration with gene therapy may be useful for tumor immunotherapy (12-14).

Type 2 recombinant AAV (rAAV2) infects dividing and non-dividing cells and maintains long-term gene expression in a number of tissues, including the liver, brain, retina and muscle $(15,16)$. Previous studies demonstrated that rAAVs are non-pathogenic in humans and exhibit low immunogenicity compared with other viral delivery systems $(17,18)$. rAAV2 viral vector expressing the glutamic acid decarboxylase (GAD) gene was successfully used to treat Parkinson's disease $(19,20)$. Those results demonstrated that rAAV2 viral vector is safer and more efficacious for human gene therapy. Therefore, rAAV2 viral vector is used to carry the human IL15 (hIL15) gene for glioblastoma therapy in this study.

Gene immunotherapy using rAAV2-bearing human IL15 gene has recently been applied in the treatment of tumors (21-23). Those studies demonstrated that rAAV2-hIL15 is 
able to express IL15 proteins and to inhibit cervical tumor and JC breast tumor growth in mice models. Previously, we determined whether rAAV2-hIL15 was able to inhibit glioblastoma growth. In the present study, rAAV2-hIL115 was succesfully produced and purified. Our results showed that rAAV2-hIL15 possesses bioactivities and is capable of delaying glioblastoma growth. Our studies may therefore provide a potential immunotherapy method for glioblastoma treatment in the future.

\section{Materials and methods}

Cell culture. DBTRG (human glioblastoma cells), HEK293 (human embryonic kidney cells), HT1080 (human fibrosarcoma cells), HT2 (murine IL2/IL15-dependent cells) and YAC-1 (murine cell line sensitive to LAK cells) cells were obtained from the Bioresource Collection and Research Center (BCRC, Shinchu, Taiwan). Cells were cultured in Dulbecco's modified Eagle's medium (DMEM) or RPMI-1640 medium supplemented with $10 \%$ fetal bovine serum and $100 \mathrm{IU} / \mathrm{ml}$ penicillin/streptomycin. Moreover, since HT2 cells are IL15dependent cells, IL15 protein (Santa Cruz Biotechnology, Inc., Santa Cruz, CA, USA) supplementation was required for maintaining survival. DMEM, RPMI, fetal bovine serum, L-glutamine, penicillin/streptomycin, sodium pyruvate and non-essential amino acids were purchased from Invitrogen (Carlsbad, CA, USA).

pAAV-hIL15 construction. The rAAV2 helper-free packing system (pAAV-MCS, pAAV-RC and pHelper plasmids) was purchased from Strategene (La Jolla, CA, USA). The human IL15 gene (hIL15) with the IL2 secretory peptide gene were kindly provided by Dr K.W. Liao (National Chiao Tung University, Hsin-Chu, Taiwan) and were amplified using the polymerase chain reaction (PCR) method. Primers containing EcoRI and BamHI sites were indicated as follows: sense primer, 5'-GAATTCAAAGAATTCATGTACAGGATGCA ACTCCT and anti-sense, 3'-GGATCCAAAGGATCCTT AAGAAGTGTTGATGAACATTTGG. The amplified hIL15 cDNA was cloned into pAAV-MCS to yield the pAAV-hIL15 plasmid.

Production and purification of rAAV2. The rAAV2 helper-free packing system was used according to the manufacturer's instructions in order to produce rAAV2-hIL15 and rAAV2-vector. Briefly, for the production of rAAV2-hIL15, HEK293 cells were cultured in fifty $15-\mathrm{cm}$ dishes and co-transfected with $2 \mathrm{mg}$ pAAV-hIL15, $2 \mathrm{mg}$ pAAV-RC and $2 \mathrm{mg}$ pAAV-helper plasmids using the $\mathrm{CaCl}_{2}$ method. For rAAV2-vector production, HEK293 cells were cultured on fifty $15-\mathrm{cm}$ dishes and co-transfected with $2 \mathrm{mg}$ pAAV-hIL15, $2 \mathrm{mg}$ pAAV-RC and $2 \mathrm{mg}$ pAAV-helper plasmids using the $\mathrm{CaCl}_{2}$ method. After $72 \mathrm{~h}$ of transfection, rAAV2-hIL15 and rAAV2-vector were produced in MEK293 cells. rAAV2-hIL15 and rAAV2-vector were purified with heparin column using a single-step column purification method and concentrated with an Amicon Ultra-15 centrifugal filter (Millipore, Billerica, MA, USA). The titer of rAAV2-hIL15 and rAAV2-vector was then measured by real-time PCR. rAAV2-hIL15 and rAAV2-vector were stored at $-80^{\circ} \mathrm{C}$ until further use.
IL15 expression assay. In order to determine IL15 protein expression, the culture media obtained from $10^{13}$ viral particles/ml rAAV2-hIL15 and rAAV2-vector-infected HT1080 cells $\left(1.5 \times 10^{5}\right.$ cells in a 6 -well plate) for 3 days were collected and determined using the ELISA assay (Biosource International, Camarillo, CA, USA). In brief, the culture media were added in 96-well plates which were coated with the hIL15 antibody. After a 4-h reaction, the plates were measured under an ELISA reader at an optical density (OD) of $450 \mathrm{~nm}$.

Bioactivity assay of rAAV2-hIL15-expressed IL15. The viability of HT2 cells was determined for the bioactivity assay of rAAV2-hIL15-expressed IL15. HT2 cells are IL15-dependent cells and IL15 is required for their survival. The culture media obtained from rAAV2-hIL15- or rAAV2- vector-infected HT1080 cells were added to HT2 cell culture. After $16 \mathrm{~h}$, the viability of HT2 cells was determined using the MTS assay (Promega, Madison, WI, USA). Media containing purified recombinant hIL15 $(1 \mu \mathrm{g} / \mathrm{ml})$ (Santa Cruz Biotechnology, Inc.) were added to HT2 cells which served as the positive control.

Cytotoxicity activity assay. Mice were infected with rAAV2-hIL15- or rAAV2-vector for >4 weeks, respectively. After the experiment mice were sacrificed, their spleens were collected and LAK cells were separated from dead cells and red blood cells by using ACK lysis buffer. YAK-1 cells are the target cells that can be killed by LAK cells. For the LAK cell cytotoxic activity assay, YAC-1 cells (target) and LAK cells (effector) were cocultured in a total volume of $200 \mu \mathrm{l}$ in RPMI-1640 containing $10 \%$ FCS in 96-well plates at various cell densities to achieve effector-to-target (E/T) ratios (12.5:1, 25:1 and 50:1) for $4 \mathrm{~h}$ at $37^{\circ} \mathrm{C}$. Target cell lysis was determined by using the lactate dehydrogenase (LDH) release assay using CytoTox $96^{\circledR}$ Non-Radioactive Cytotoxicity assay kit (Promega) and was calculated as: (OD490 nm of sample - OD490 nm with spontaneous release of LDH from target cells - OD490 nm with spontaneous release of LDH from effector cells) x100/(OD490 nm with maximum release of LDH from target cells - OD490 nm with spontaneous release of LDH from effector cells).

Animal studies. Female nude mice (4 weeks old) were obtained from the National Animal Laboratory Center (Taipei, Taiwan). Every 8 nude mouse was infected on one site of the quadriceps muscles with rAAV2-hIL15 (5x10 ${ }^{12}$ viral particles), rAAV2vector ( $5 \times 10^{12}$ viral particles), and PBS (mock), respectively. After 1 month, $10^{7}$ DBTRG glioblastoma cells were transplanted on the lower abdominal region of the mice. Tumor growth was observed every 4 days, the tumor volume was measured with the caliper and calculated as $1 / 2 \mathrm{x}$ length $\mathrm{x}$ width ${ }^{2}$. Animals were sacrificed when tumor size was $1,500 \mathrm{~mm}^{3}$.

Statistical analysis. Data are presented as the mean \pm SEM. Statistical significance was analyzed using the Student's t-test. Survival analysis was performed using the Kaplan-Meier method. $\mathrm{P}<0.05$ was considered statistically significant.

\section{Results}

rAAV2-hIL15 expresses hIL15 protein with bioactivity in vitro. HT1080 cells were treated with rAAV2-hIL15, rAAV2-vector 


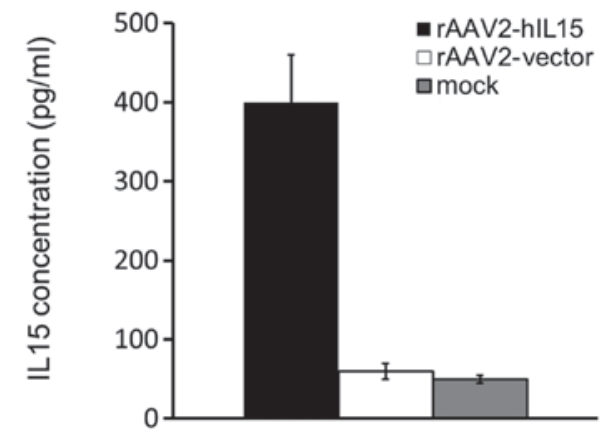

Figure 1. hIL15 protein expression. Culture media obtained from rAAV2hIL15, rAAV2-vector and non-infected (PBS-treated mock) HT1080 cells were measured using ELISA. Notably, only the culture media obtained from rAAV2-hIL15-infected HT1080 cells exhibited high hIL15 level. hIL15, human interleukin 15; rAAV2, recombinant adeno-associated virus serotype 2; PBS, phosphate-buffered saline; ELISA, enzyme-linked immunosorbent assay.

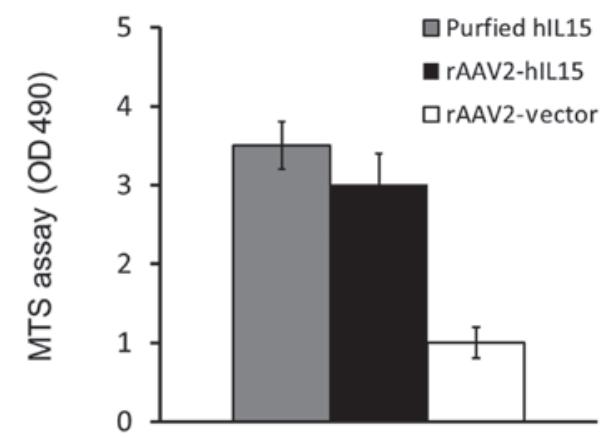

Figure 2. Bioactivity of hIL15 in vitro. Culture media obtained from the rAAV2-hIL15 and rAAV2-vector-infected HT1080 cells were added to HT2 cell culture for the bioactivity assay. Purified hIL15 was added to HT2 cell culture as the positive control. Bioactivity was determined by HT2 cell viability, measured using the MTS assay. Notably, the rAAV2-hIL15 group had high cell viability, similar to the purified hIL15 group. hIL15, human interleukin 15; rAAV2, recombinant adeno-associated virus serotype 2 .

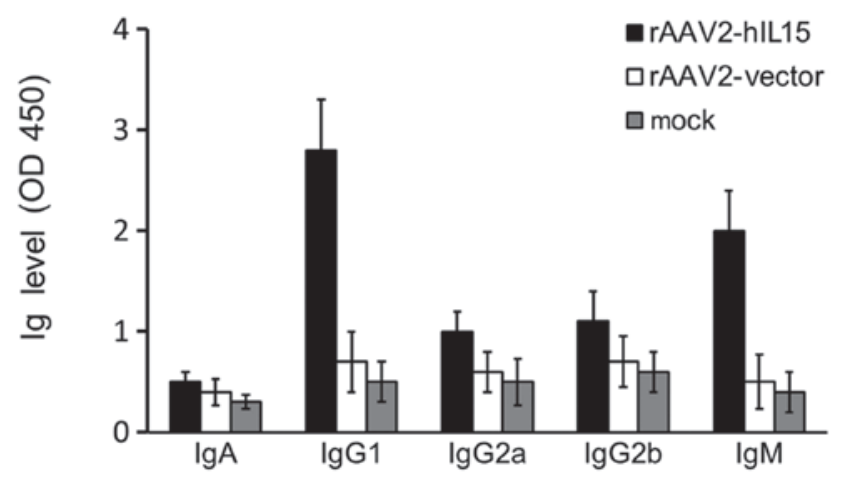

Figure 3. Immunoglobulin (Ig) level was analyzed among rAAV2-hIL15treated, rAAV2-vector-treated and mock groups. Sera obtained from these groups were examined, respectively. Quantification of immunoglobulin levels was measured via ELISA. hIL15, human interleukin 15; rAAV2, recombinant adeno-associated virus serotype 2; ELISA, enzyme-linked immunosorbent assay.

and PBS (mock), respectively. After $72 \mathrm{~h}$, the media obtained from the above groups were determined for the hIL15 level using ELISA. Our data showed that rAAV2-hIL15-treated HT1080

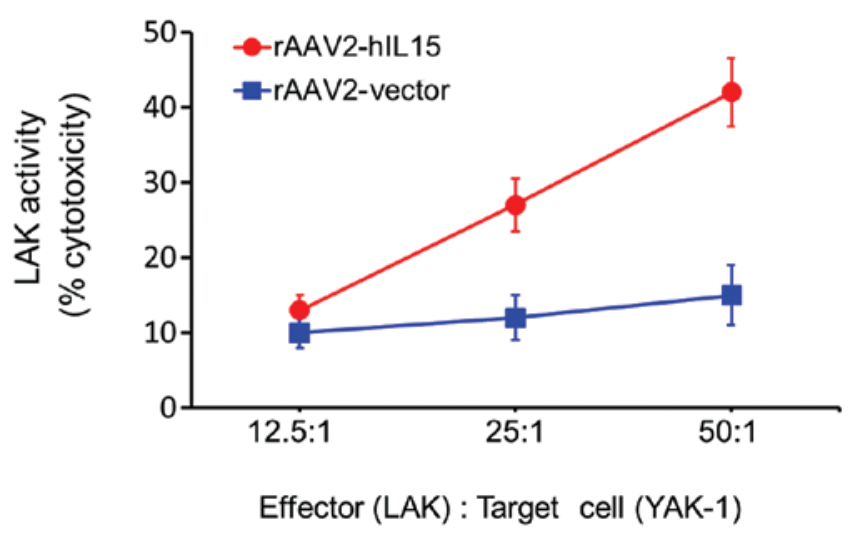

Figure 4. rAAV2-hIL15 induced cytotoxic activity of LAK cells. LAK cells obtained from rAAV2-hIL15-treated (red) and rAAV2-vector-treated groups (blue) were cocultured with their target cells (YAK-1 cells) at various ratios, respectively. Cytotoxtic activity of LAK cells was measured using the LDH release assay. hIL15, human interleukin 15; rAAV2, recombinant adenoassociated virus serotype 2; LAK, lymphokine-activated killer.

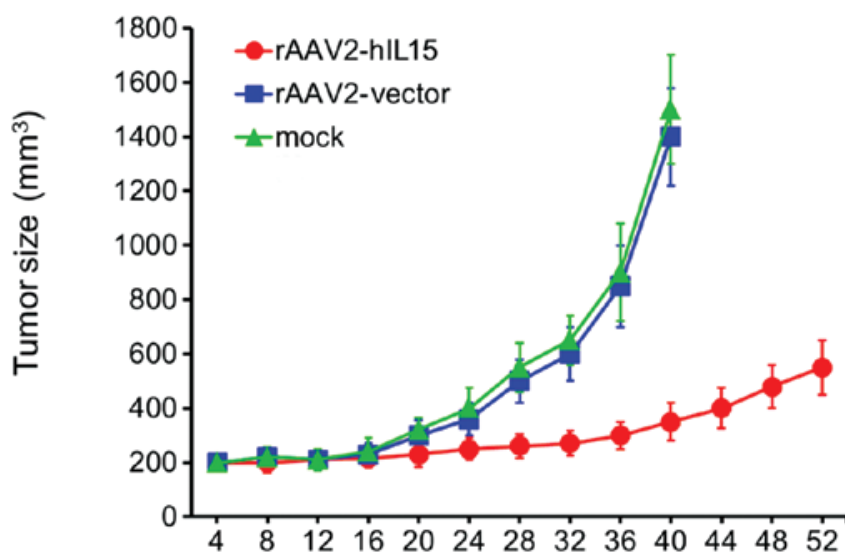

Day after tumor inoculation

Figure 5. rAAV2-hIL15 effectively delayed glioblastoma tumor growth Tumor growth was observed every 4 days. Tumor size was calculated as $1 / 2 \mathrm{x}$ length $\mathrm{x}$ width ${ }^{2}$ and presented as the mean \pm SEM. Notably, tumor size was not significantly different between the rAAV2-vector (blue) and mock (green) groups. Tumor growth was significantly slow in the rAAV2-hIL15 group (red). hIL15, human interleukin 15; rAAV2, recombinant adenoassociated virus serotype 2; SEM, standard error of the mean.

cells expressed a 4- to 5-fold level of hIL15 protein as compared with mock or rAAV2-vector groups (Fig. 1). We further determined the bioactivity of rAAV2-hIL15-expressed hIL15 in vitro using a cell viability assay. HT2 cells are IL15-dependent cells that survive under IL15 supplementation. In this study, culture media obtained from the rAAV2-hIL15 and rAAV2-vector were added to HT2 cells for $48 \mathrm{~h}$, and HT2 cell viability was determined by using the MTS assay. Our results demonstrated that the media obtained from the rAAV2-hIL15 group showed higher HT2 cell viability as compared with that of the rAAV2-vector group. The purified hIL15 protein served as the positive control (Fig. 2). Thus, results showed that rAAV2-hIL15 expressed hIL15 protein with bioactivity in vitro. 
rAAV2-hIL15 induces the production of IgG1 and IgM in nude mice. Nude mice were injected with rAAV2-hIL15, rAAV2-vector and mock in the quadriceps muscle of the left thigh, respectively. After 28 days, the mice sera obtained from the above three groups were analyzed for immunoglobulin (Ig) production (IgG1, IgG2a, IgG2b, IgM and IgA). IgG1 and IgM levels were higher in the rAAV2-hIL15 group compared with the rAAV2-vector and mock groups (Fig. 3). However, the levels of IgG2a, IgG2b and IgA were not significantly different among the rAAV2-hIL15, rAAV2-vector and mock groups. Therefore, our data showed that rAAV2-hIL15 was capable of inducing the production of $\mathrm{IgG1}$ and $\mathrm{IgM}$ in nude mice.

rAAV2-hIL15 activates cytotoxic activity of LAK cells. Mice were administered an intramuscular injection over the quadriceps muscle of the left thigh with rAAV2-hIL15 and rAAV2-vector, respectively, for 28 days. Consequently, the mice were sacrificed and their spleens collected. LAK cells were then separated and collected from dead cells and red blood cells. YAK-1 cells, the target cells of LAK cells, were used for the cytotoxic activity assay of LAK cells. YAK-1 and LAK cells were cocultured according to different ratios and the cytotoxic activity of LAK cells was studied using CytoTox $96{ }^{\circledR}$ Non-Radioactive Cytotoxicity assay (LDH release assay). The cytotoxic activity of LAK cells was significantly induced in the rAAV2-hIL15 group (Fig. 4). At an effector/target cell ratio of 50:1, the cytotoxic activity level of LAK cells was induced with rAAV2-hIL15 up to 3-fold as compared with the rAAV2-vector group. This result suggested that rAAV2-hIL15 was able to activate the cytotoxic activity of LAK cells.

rAAV2-hIL15 delays efficient glioblastoma growth. After nude mice were intramuscularly injected with rAAV2-hIL15, rAAV2-vector and mock, respectively, for 28 days, mice were with human glioblastoma DBTRG cells subcutaneously injected. Tumor size was determined every 4 days. The tumor growth rate was slower in the rAAV2-hIL15 group compared with that in the rAAV2-vector and mock groups (Fig. 5). Although there was still a slow-paced tumor growth in the rAAV2-hIL15 group, rAAV2-hIL15 delayed tumor growth as compared with the control groups.

\section{Discussion}

Treatment of human malignant brain tumor remains a challenge and the average survival time of brain tumor patients is $\sim 12-24$ months when traditional therapies are employed $(1,24,25)$. Thus, new therapies for brain tumor patients have been suggested and studied (26-28). Currently, gene therapy is also used in the treatment of brain tumor (28-30). Adeno-associated virus (AAV), lentivirus, herpes virus and adenovirus transfer are generally applied in gene therapy $(31,32)$. Due to the non-pathogenic characteristics and the low immunogenicity of rAAV2, rAAV2 is considered safer than other viral delivery systems (33-36). However, rAAV2 is a small virus and it only carries small genes of $\sim 3,000$ nucleotides (37). Findings of previous studies have indicated that IL15 has a short half-life and repeated daily injection is required for immunotherapy in clinical course (9-11). Therefore, gene therapy is suggested for IL15 application.
Since the human IL15 gene is $~ 600$-nucleotide long, it can be carried by rAAV2 viral delivery systems. In this study, we successfully produced rAAV2-hIL15 and revealed that rAAV2-hIL15 possesses antitumor bioactivities in a xenografted brain tumor mice model.

A previous study demonstrated that rAAV2-hIL15 was able to induce $\operatorname{IgG} 1, \operatorname{IgG} 2 \mathrm{~b}$ and $\mathrm{IgM}$ production in a nude mice model (23). However, IgG2b level was lower than that of IgG1 and IgM. Concerning our results, rAAV2-hIL15 was able to enhance IgG1 and IgM production, but there was no significant difference in the IgG2b level between the rAAV2-hIL15 group and control groups (Fig. 3). A comparison of our results with those of this previously published study suggests that rAAV2-hIL15 is capable of inducing IgG1 and IgM production although it does not induce substantial IgG2b production. Recently, it has been demonstated that rAAV2-hIL15 is able to activate LAK cell cytotoxic activity (21-23). Our data also showed that rAAV2-hIL15 induces LAK cell cytotoxic activity in glioblastoma cells.

It has been suggested that rAAV2-hIL15 inhibits tumor growth on both human cervical tumors and mouse JC breast tumors (21-23). Additionally, rAAV2-hIL15 also inhibits the growth of human glioblastoma cells. Taken together, these studies suggest that rAAV2-hIL15 inhibits the tumor growth of various tumor types. Recently, human interleukin 12 (hIL12) was carried by rAAV2 for the treatment of brain tumor (38). This study has demonstrated that rAAV2-hIL12 induces Ig production and cytotoxic activity of LAK cells, and exerts antitumor activity on brain tumor. Ig production, the cytotoxic activity of LAK cells and the antitumor activity on human glioblastoma have also been demonstrated in this study by using rAAV2-hIL15. However, Ig production between rAAV2hIL12 and rAAV2-hIL15 is different. IgG1 and IgG2a levels were significantly increased previously by rAAV2-hIL12 treatment (38). However, IgG1 and IgM levels were significantly increased by rAAV2-hIL15 treatment in our study. Although there are different Ig production levels between rAAV2-hIL12 and rAAV2-hIL15, they are able to inhibit brain tumor growth effectively. Therefore, we suggest that the rAAV2 gene transfer system is a useful method for tumor immunotherapy and a potential therapeutic method that may be combined with hIL12 and hIL15 for brain tumor immunotherapy in the future.

\section{Acknowledgements}

This study was supported by the National Science Council of Taiwan (nos. NSC99-2320-B-039-030-MY3, NSC99-2632-B039-001-MY3 and NSC100-2321-B-039-004 to YLY) and the University of Texas MD Anderson-China Medical University Hospital Sister Institution Fund (DMR-101-115 to YLY as well as NSC101-2311-B-039-001 and CMU100-N2-09 to RHC).

\section{References}

1. Daneyemez M, Gezen F, Canakci Z and Kahraman S: Radical surgery and reoperation in supratentorial malignant glial tumors. Minim Invasive Neurosurg 41: 209-213, 1998.

2. Stephenson KB, Barra NG, Davies E, Ashkar AA and Lichty BD: Expressing human interleukin-15 from oncolytic vesicular stomatitis virus improves survival in a murine metastatic colon adenocarcinoma model through the enhancement of anti-tumor immunity. Cancer Gene Ther 19: 238-246, 2012. 
3. McBride WH: Integration of adenovirus thymidine kinase suicide-gene therapy with surgery and radiation therapy for malignant glioma. Future Oncol 8: 17-20, 2012.

4. Srivastava D, Joshi G, Somasundaram K and Mulherkar R: Mode of cell death associated with adenovirus-mediated suicide gene therapy in HNSCC tumor model. Anticancer Res 31: 3851-3857, 2011.

5. Munger W, DeJoy SQ, Jeyaseelan R Sr, et al: Studies evaluating the antitumor activity and toxicity of interleukin-15, a new $\mathrm{T}$ cell growth factor: comparison with interleukin-2. Cell Immunol 165: 289-293, 1995.

6. Carson WE, Giri JG, Lindemann MJ, et al: Interleukin (IL) 15 is a novel cytokine that activates human natural killer cells via components of the IL-2 receptor. J Exp Med 180: 1395-1403, 1994.

7. Mrozek E, Anderson P and Caligiuri MA: Role of interleukin-15 in the development of human $\mathrm{CD} 56^{+}$natural killer cells from $\mathrm{CD} 4^{+}$hematopoietic progenitor cells. Blood 87: 2632-2640, 1996.

8. Sparano JA, Fisher RI, Weiss GR, et al: Phase II trials of high-dose interleukin-2 and lymphokine-activated killer cells in advanced breast carcinoma and carcinoma of the lung, ovary, and pancreas and other tumors. J Immunother Emphasis Tumor Immunol 16: 216-223, 1994.

9. Chapoval AI, Fuller JA, Kremlev SG, Kamdar SJ and Evans R: Combination chemotherapy and IL15 administration induce permanent tumor regression in a mouse lung tumor model: NK and $\mathrm{T}$ cell-mediated effects antagonized by B cells. J Immunol 161: 6977-6984, 1998.

10. Lasek W, Basak G, Switaj T, et al: Complete tumour regressions induced by vaccination with IL-12 gene-transduced tumour cells in combination with IL15 in a melanoma model in mice. Cancer Immunol Immunother 53: 363-372, 2004.

11. Rubinstein MP, Kadima AN, Salem ML, Nguyen CL, Gillanders WE and Cole DJ: Systemic administration of IL15 augments the antigen-specific primary $\mathrm{CD} 8^{+} \mathrm{T}$ cell response following vaccination with peptide-pulsed dendritic cells. J Immunol 169: 4928-4935, 2002.

12. Croce M, Meazza R, Orengo AM, et al: Sequential immunogene therapy with interleukin-12- and interleukin-15-engineered neuroblastoma cells cures metastatic disease in syngeneic mice. Clin Cancer Res 11: 735-742, 2005.

13. Suzuki K, Nakazato H, Matsui H, et al: NK cell-mediated antitumor immune response to human prostate cancer cell, PC-3: immunogene therapy using a highly secretable form of interleukin-15 gene transfer. J Leukoc Biol 69: 531-537, 2001.

14. Kimura K, Nishimura H, Hirose K, Matsuguchi T, Nimura Y and Yoshikai Y: Immunogene therapy of murine fibrosarcoma using IL15 gene with high translation efficiency. Eur J Immunol 29: 1532-1542, 1999.

15. Ponnazhagan S, Curiel DT, Shaw DR, Alvarez RD and Siegal GP: Adeno-associated virus for cancer gene therapy. Cancer Res 61 6313-6321, 2001.

16. Xiao X, Li J and Samulski RJ: Efficient long-term gene transfer into muscle tissue of immunocompetent mice by adeno-associated virus vector. J Virol 70: 8098-8108, 1996.

17. Jennings K, Miyamae T, Traister R, et al: Proteasome inhibition enhances AAV-mediated transgene expression in human synoviocytes in vitro and in vivo. Mol Ther 11: 600-607, 2005.

18. Jooss K, Yang Y, Fisher KJ and Wilson JM: Transduction of dendritic cells by DNA viral vectors directs the immune response to transgene products in muscle fibers. J Virol 72: 4212-4223, 1998.

19. Emborg ME, Carbon M, Holden JE, et al: Subthalamic glutamic acid decarboxylase gene therapy: changes in motor function and cortical metabolism. J Cereb Blood Flow Metab 27: 501-509, 2007.
20. Kaplitt MG, Feigin A, Tang C, et al: Safety and tolerability of gene therapy with an adeno-associated virus (AAV) borne GAD gene for Parkinson's disease: an open label, phase I trial. Lancet 369: 2097-2105, 2007.

21. Yu YL, Wei CW, Chen YL, Chen MH and Yiang GT: Immunotherapy of breast cancer by single delivery with rAAV2-mediated interleukin-15 expression. Int J Oncol 36: 365-370, 2010.

22. Yiang GT, Chou PL, Tsai HF, et al: Immunotherapy for SV40 T/t antigen-induced breast cancer by recombinant adeno-associated virus serotype 2 carrying interleukin- 15 in mice. Int J Mol Med 29: 809-814, 2012.

23. Yiang GT, Harn HJ, Yu YL, et al: Immunotherapy: rAAV2 expressing interleukin-15 inhibits HeLa cell tumor growth in mice. J Biomed Sci 16: 47, 2009.

24. Kayama T, Kumabe T, Tominaga T and Yoshimoto T: Prognostic value of complete response after the initial treatment for malignant astrocytoma. Neurol Res 18: 321-324, 1996.

25. Kowalczuk A, Macdonald RL, Amidei C, et al: Quantitative imaging study of extent of surgical resection and prognosis of malignant astrocytomas. Neurosurgery 41: 1028-1038, 1997.

26. Li YM and Hall WA: Targeted toxins in brain tumor therapy. Toxins (Basel) 2: 2645-2662, 2010.

27. Ahmed AU, Ulasov IV, Mercer RW and Lesniak MS: Maintaining and loading neural stem cells for delivery of oncolytic adenovirus to brain tumors. Methods Mol Biol 797: 97-109, 2012.

28. Kim CY, Park SH, Jeong M, et al: Preclinical studies for pharmacokinetics and biodistribution of Ad-stTRAIL, an adenovirus delivering secretable trimeric TRAIL for gene therapy. Exp Mol Med 43: 580-586, 2011.

29. Lee EX, Lam DH, Wu C, et al: Glioma gene therapy using induced pluripotent stem cell derived neural stem cells. Mol Pharm 8: 1515-1524, 2011.

30. King GD, Muhammad AK, Larocque D, et al: Combined Flt3L/TK gene therapy induces immunological surveillance which mediates an immune response against a surrogate brain tumor neoantigen. Mol Ther 19: 1793-1801, 2011.

31. Heilbronn R and Weger S: Viral vectors for gene transfer: current status of gene therapeutics. Handb Exp Pharmacol: 143-170, 2010.

32. Lundstrom K: Gene therapy applications of viral vectors. Technol Cancer Res Treat 3: 467-477, 2004.

33. Wang L, Calcedo R, Nichols TC, et al: Sustained correction of disease in naive and AAV2-pretreated hemophilia B dogs: AAV2/8-mediated, liver-directed gene therapy. Blood 105: 30793086,2005

34. Flannery JG,Zolotukhin S, Vaquero MI,LaVail MM, Muzyczka N and Hauswirth WW: Efficient photoreceptor-targeted gene expression in vivo by recombinant adeno-associated virus. Proc Natl Acad Sci USA 94: 6916-6921, 1997.

35. Snyder RO and Flotte TR: Production of clinical-grade recombinant adeno-associated virus vectors. Curr Opin Biotechnol 13: 418-423, 2002

36. Li SF, Wang RZ, Meng QH, et al: Intra-ventricular infusion of rAAV1-EGFP resulted in transduction in multiple regions of adult rat brain: a comparative study with rAAV2 and rAAV5 vectors. Brain Res 1122: 1-9, 2006.

37. Dong JY, Fan PD and Frizzell RA: Quantitative analysis of the packaging capacity of recombinant adeno-associated virus. Hum Gene Ther 7: 2101-2112, 1996.

38. Chiu TL, Lin SZ, Hsieh WH and Peng CW: AAV2-mediated interleukin-12 in the treatment of malignant brain tumors through activation of NK cells. Int J Oncol 35: 1361-1367, 2009. 